\title{
OTR8 Optimized production of monoclonal antibody used in Flavivirus immunoassays for different projects of vaccine development
}

Raquel de Souza Martins ${ }^{1}$, Carolina dos Reis Albuquerque Cajaraville ${ }^{2}$, Fernando de Paiva Conte ${ }^{1}$, Márcia Arissawa ${ }^{1}$

1 Laboratório de Tecnologia de Anticorpos Monoclonais, BioManguinhos, Fundação Oswaldo Cruz, Rio de Janeiro, Brazil

2 Laboratório de Tecnologia Virológica, Bio-Manguinhos, Fundação Oswaldo Cruz, Rio de Janeiro, Brazil

Introduction: D1-4G2-4-15 hybridoma is responsible for secreting a monoclonal antibody (mAb) against Flavivirus group antigen and is currently used in different projects of vaccine development at BioManguinhos. Despite considerable progress in cell culture techniques, fetal bovine serum (FBS) supplemented media are still commonly used during hybridoma growth and maintenance in vitro. However, its use is controversial due to batch-to-batch composition variations, increased safety risk, low mAb yields after purification steps and ethical aspects related to the use of animals. In this context, adaptation of the hybridoma culture to serum-free medium meets regulatory concerns regarding the safety of animal-derived products.

Objective: To develop an optimized process using serum-free medium for production of mAb used in Flavivirus immunoassays for different projects of vaccine development.

Methodology: Hybridoma D1-4G2-4-15 was cultivated in roller bottles using high glucose DMEM supplemented with $10 \%$ FBS and $6.4 \mathrm{mM} \mathrm{L-}$ glutamine (DMEM/FBS10\%). In another set of experiments, the hybridoma cultivated in roller bottles with DMEM/SFB10\% was weaned directly in serum-free medium EX-CELL/6.4 mM glutamine, until considered adapted (viability $>90 \%$ for, at least, three cell passages with cells in logarithmic phase). Cell counts were performed in Neubauer chamber, under optical microscope, after dilution in trypan blue $0.4 \%$. Each sample was centrifuged (200 g, $10 \mathrm{~min}$ ) and the supernatant was used for determination of murine IgG (Easy-Titer Antibody Assay kit, Pierce), L-glutamine (YSY 2700 analyzer) and Llactate (Roche commercial kit). Specific cell growth rate $(\mu)$ and doubling time $(\mathrm{dt})$ were calculated using the differential method, during the exponential growth phase. Specific L-glutamine consumption rate (qSglu), and specific L-lactate and IgG production rates (qPlac and qPIgG, respectively) were estimated by plotting total cell concentration, cumulative substrate consumption or production, versus the integral of viable cells and fitting the plots with a regression coefficient of close to one. Foci reduction neutralization test (FRNT) using Vero cells infected with Dengue virus serotypes 1, 2, 3, 4 and Yellow Fever virus FA17DD was used to confirm mAb titer and specificity. 
Results: The maximum hybridoma viable cell concentration achieved in DMEM/FBS $10 \%$ was $3.4 \times 106$ cells $/ \mathrm{ml}$; with $\mu=0.035 \mathrm{~h}-1$, $\mathrm{dt}=19.8 \mathrm{~h}$,

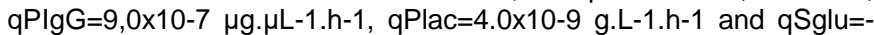
3.0x10-9 g.L-1.h-1. D1-4G2-4-15 direct serum-free adaptation was achieved after 15 passages in EX-CELL, with maximum viable cell concentration of $2.8 \times 106$ cells $/ \mathrm{ml}$; with $\mu=0.033 \mathrm{~h}-1$, dt $=24.4 \mathrm{~h}, \mathrm{qPIgG}$ $=5.0 \times 10-7, \mathrm{qPlac}=4.0 \times 10-9$ and $\mathrm{qSglu}=-4.0 \times 10-9 . \mathrm{mAb}$ produced in DMEM FBS10\% specifically recognized Dengue-1,-2,-3,-4 and FA17DD virus as commercial available antibody. Further experiments will be performed to check mAb produced in EX-CELL.

Conclusion: The results showed that although serum-free adapted hybridoma had a slower growth and metabolism rate, as expected, its IgG production capacity was preserved. Thus, we provide a more controlled and standardized cell culture environment for mAb production.

Keywords: Fetal Bovine Serum, Hybridoma, Antibodies 